\title{
Some Closed-Form Solutions of the Temperature Field due to Bending Magnet and Undulator Heating in APS ${ }^{1}$
}

I. C. Sheng and T. Nian Argonne National Laboratory 9700 South Cass Ave. Argonne, IL 60439

\begin{abstract}
U. S. Government purpose:

Several temperature field solutions due to bending magnet and undulator x-ray heating are devlobed and presented in this paper. The Gaussian power distribution is simulated as the bending magnet whereas a Gaussian-parabolic type of power distribution is used for the undulator/wiggler heating. The heating on a two-dimensional plane, three-dimensional block, thin disk, infinite wedge plane, infinite wedge block, and beryllium-copper composite are analyzed. Parametric studies are also included to determine the optimized temperature.
\end{abstract}

The submitted manuzitript has been outhored by contrector of 8 the U.S. Governomint under contrace No. W:3trosens-38. Accordingly, the U.S. Government retains:

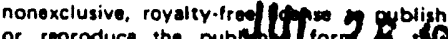

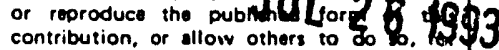

\section{Nomenclature}

$\begin{aligned} B & \text { Magnetic field } & q & \text { Power distribution function } \\ q_{o} & \text { Peak power } & \sigma_{o} & \text { Standard deviation } \\ E & \text { Positron energy } & I & \text { Beam current } \\ l & \text { Distance from the source } & N & \text { Number of periods } \\ \sigma_{z} & \text { Parabolic deviation } & T & \text { Temperature } \\ k & \text { Conductivity } & t & \text { Thickness of the material } \\ a, b & \text { Width and length } & h_{c} & \text { Convection coefficient } \\ \rho & \text { Material density } & c & \text { Specific heat } \\ G^{*} & \text { Green function } & \theta_{o} & \text { Wedge angle } \\ \alpha_{t} & \text { Absorption coefficient } & T_{\infty} & \text { Ambient temperature } \\ B i & \text { Biot number } & x_{o} & \text { Center location of the beam } \\ r_{o} & \text { radius } & P_{T} & \text { Total undulator/wiggler power } \\ x, y, z & \text { Cartesian coordinate } & r, \theta, z & \text { Cylindrical coordinate }\end{aligned}$

Subscripts $C u$ and $B e$ indicate copper and beryllium, respectively.

\section{Introduction}

The Advanced Photon Source (APS) is a 7-GeV synchrotron accelerator which will produce a high-powered beam of $\mathbf{x}$-rays. Several components such as crotch absorbers, wedge absorbers, and photon shutters are constantly heated by the beam. In addition, as a result of beam missteering, $x$-rays might accidentally hit the storage ring and cause thermomechanical instability problems. Therefore, the determination of the temperature field either in steady state or transient state is important. The bending magnet fan can be simulated as a two-dimensional Gaussian type power distribution whereas the undulator/wiggler, the Gaussian-parabolic, or circular Gaussian power depend on the maximum deflection parameter $K$. Because of the characteristic of these beam power profiles, sume analytical temperature solutions are available and have been solved [1, 2, 3]. In this paper, five other analytical solutions are presented and can be used as a parametric studies when they are applied to synchrotron heating problems.

The bending magnet power can be written as [4]

$$
q\left[\frac{K W}{\text { area }}\right]=\frac{5.425 E^{4}[G e V] B[T] I[m A]}{l^{2}} \exp \left(-\frac{1957 E y^{2}}{0.608 l}\right)=q_{0} \exp \left(-\frac{y^{2}}{2 \sigma_{0}^{2}}\right) .
$$

\footnotetext{
${ }^{1}$ Work supported by U.S. Department of Energy, Office of Basic Energy Sciences under Contract No. W-31-109-E:VG-38.
}

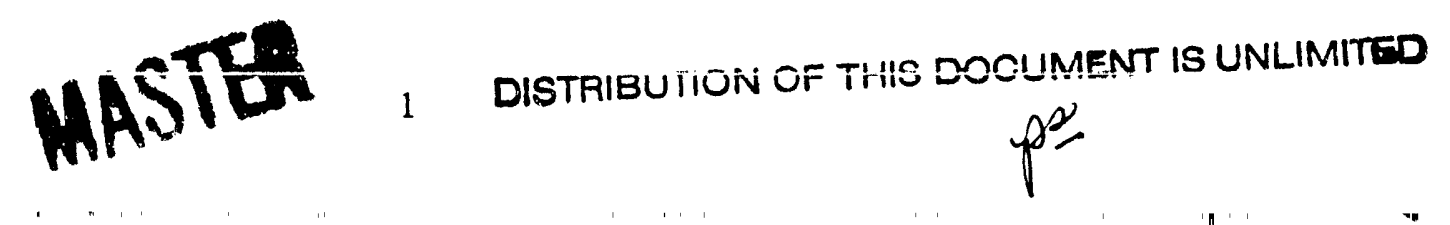


If the maximum deflection parameter $K=1$, the undulator beam can be expressed as a circular Gaussian power [5], that is,

$$
q\left[\frac{K W}{\text { area }}\right]=\frac{10.84 E^{4}[\mathrm{GeV}] B_{o}[T] I[m A] N G}{l^{2}} \exp \left(-\frac{1957 E r^{2}}{0.608 l}\right)=q_{o} \exp \left(-\frac{r^{2}}{2 \sigma_{o}^{2}}\right) .
$$

If $K>1$ the undulator/wiggler is better fitted as a Gaussian-parabolic distribution

$$
q=q_{\circ} \exp \left(-\frac{y^{2}}{2 \sigma_{o}^{2}}\right)\left(1-\frac{x^{2}}{2 \sigma_{z}^{2}}\right)
$$

where $q_{\circ}$ and $\sigma_{o}$ are identical to Eq. (2). Knowing the total power $P_{T}[K W]=6.33 \times 10^{-4} E^{2}[G e V] B_{o}^{2}[T] L[m] I$ $[m A], \sigma_{z}$ is defined as

$$
\sigma_{z}=\frac{3 P_{T}}{8 q_{o} \sqrt{\pi} \sigma_{o}}
$$

\section{Rectangular Plate, $0 \leq x \leq a, 0 \leq y \leq b$ with Gaussian Power on $y=0, x=x_{o}$ and Cooling on the Other Side}

The governing equation reads

$$
\frac{\partial^{2} \bar{T}}{\partial \xi^{2}}+\frac{\partial^{2} \bar{T}}{\partial \eta^{2}}=0
$$

As shown in Fig. 1.a, the boundary conditions are written as

$$
\begin{aligned}
& \frac{\partial \bar{T}}{\partial \eta}(\xi, 0)=-\exp \left(-\frac{\left(\xi-\xi_{o}\right)^{2}}{\sigma_{\xi}^{2}}\right), \\
& \frac{\partial \bar{T}}{\partial \eta}(\xi, 1)=-B i \bar{T}
\end{aligned}
$$

and

$$
\frac{\partial \bar{T}}{\partial \xi}(0, \eta)=\frac{\partial \bar{T}}{\partial \xi}(\alpha, \eta)=0
$$

where

$$
\bar{T}=\frac{k\left(T-T_{\infty}\right)}{q_{o} t}, \quad \alpha=\frac{a}{t}, \quad B i=\frac{h_{c} t}{k}, \quad \xi=\frac{x}{t}, \quad \xi_{o}=\frac{x_{o}}{t}, \quad \eta=\frac{y}{t}, \quad \sigma_{\xi}=\frac{\sqrt{2} \sigma_{o}}{t} .
$$

By employing the separation variable method, solution $\bar{T}$ becomes

$$
\bar{T}=C_{\circ}\left[\eta-1-\frac{1}{B i}\right]+\sum_{m=0}^{\infty} C_{m} \exp \left(-\lambda_{m} \eta\right) \cos \left(\lambda_{m} \xi\right)\left[1+\frac{\lambda_{m}-B i}{\lambda_{m}+B i} \exp \left(2 \lambda_{m}(\eta-1)\right)\right],
$$

where $\lambda_{m}=\frac{m \pi}{\alpha}$ and the maximum temperature can be written as

$$
\bar{T}=-C_{0}\left[1+\frac{1}{B i}\right]+\sum_{m=0}^{\infty} C_{m}\left[1+\frac{\lambda_{m}-B i}{\lambda_{m}+B i} \exp \left(-2 \lambda_{m}\right)\right]
$$


where

$$
C_{o}=-\frac{1}{\alpha} \int_{0}^{\alpha} \exp \left(-\frac{\left(\xi-\xi_{0}\right)^{2}}{\sigma_{\xi}^{2}}\right) d \xi=-\frac{\sqrt{\pi} \sigma_{\xi}}{2 \alpha}\left[\operatorname{erf}\left(\frac{\alpha-\xi_{0}}{\sigma_{\xi}}\right)+\operatorname{erf}\left(\frac{\xi_{0}}{\sigma_{\xi}}\right)\right]
$$

and

$$
\begin{aligned}
C_{m}= & -\frac{2 \int_{o}^{\alpha} \exp \left(-\frac{\left(\xi-\xi_{o}\right)^{2}}{\sigma_{\xi}^{2}}\right) \cos \left(\lambda_{m} \xi\right) d \xi}{\lambda_{m} \alpha\left(-1+\exp \left(-2 \lambda_{m}\right)\left(\frac{\lambda_{m}-B i}{\lambda_{m}+B i}\right)\right)}=-\frac{\sqrt{\pi} \sigma_{\xi} \exp \left(-\frac{\lambda_{m} \sigma_{\xi}^{2}}{4}\right)}{\lambda_{m} \alpha\left(-1+\exp \left(-2 \lambda_{m}\right)\left(\frac{\lambda_{m}-B i}{\lambda_{m}+B i}\right)\right)} \times \\
& {\left[\operatorname{Re}\left(\exp \left(-\lambda_{m} \xi_{o} i\right)\left(\operatorname{erf}\left(\frac{2 \alpha-2 \xi_{\circ}+\lambda_{m} \sigma_{\xi}^{2} i}{2 \sigma_{\xi}}\right)\right)-\operatorname{erf}\left(\frac{-2 \xi_{\circ}+\lambda_{m} \sigma_{\xi}^{2} i}{2 \sigma_{\xi}}\right)\right)\right] . }
\end{aligned}
$$

If the Gaussian power is placed at the origin, i.e., $\xi_{0}=0$, then it is equilibrium to a rectangular block $2 a \times b$ and the power is heating at the center of the width $a$; thus the coefficients $C_{o}$ and $C_{m}$ yield

$$
\lim _{\xi_{o} \rightarrow 0} C_{o}=-\frac{\sqrt{\pi} \sigma_{\xi}}{2 \alpha} \operatorname{erf}\left(\frac{\alpha}{\sigma_{\xi}}\right), \lim _{\xi_{o} \rightarrow 0} C_{m}=\frac{-\sqrt{\pi} \sigma_{\xi} \exp \left(-\frac{\lambda_{m} \sigma_{\xi}^{2}}{4}\right)}{\lambda_{m} \alpha\left(-1+\exp \left(-2 \lambda_{m}\right) \frac{\lambda_{m}-B i}{\lambda_{m}+B i}\right)} \operatorname{Re}\left[\operatorname{erf}\left(\frac{2 \alpha+\lambda_{m} \sigma_{\xi}^{2} i}{2 \sigma_{\xi}}\right)\right](4)
$$

which is identical to the results described in [6].

Figure 2 shows the parametric study of a copper block heated by a Gaussian heat flux with $b=4 \mathrm{~cm}$, peak heat flux $q_{o}=260 \frac{\mathrm{W}}{\mathrm{mm}^{2}}$, standard deviation $\sigma_{o}=0.009 \mathrm{~cm}$, and $\xi_{\circ}=\frac{b}{2}$. By changing the width $a$ and convection $h_{c}$, the maximum temperature on the heating surface (Fig. 2.a) reveals that when the convection coefficient is less than $3 \frac{W}{\mathrm{~cm}^{2} \mathrm{oC}}$, the temperature rise is approximately linearly proportional to $h_{c}$ as $a>0.3 \mathrm{~cm}$ and is independent of the width change. This is because the material has reached its conduction limit due to the highly concentrated beam power. On the other hand, as $a<0.1 \mathrm{~cm}$, the temperature increases dramatically and is independent of the convection coefficient. Fig. 2.b shows the maximum temperature occurs on the water tube $(y=t)$, and the temperature decreases depending on the combination of the thickness and water convection coefficient. This solution is applicable to crotch absorbers, wedge absorbers, end absorbers, photon shutters, and fixed masks.

\section{Rectangular Block $-a / 2 \leq x \leq a / 2,-b / 2 \leq y \leq b / 2,-t \leq z \leq 0$ with Gaussian-Parabolic Power on $z=0$ and Cooling on the Other Side}

The governing equation reads

$$
\frac{\partial^{2} \bar{T}}{\partial \xi^{2}}+\frac{\partial^{2} \bar{T}}{\partial \eta^{2}}+\frac{\partial^{2} \bar{T}}{\partial \gamma^{2}}=0
$$

and the boundary conditions

$$
\frac{\partial \bar{T}}{\partial \gamma}(\xi, \eta, 0)=-\exp \left(-\frac{\xi^{2}}{2 \alpha^{2}}\right)\left(1-\frac{\eta^{2}}{2 \beta^{2}}\right), \quad \frac{\partial \bar{T}}{\partial \gamma}(\xi, \eta, 1)=-B i \bar{T}
$$

where

$$
\bar{T}=\frac{k\left(T-T_{\infty}\right)}{q_{o} t}, \quad \xi=\frac{x}{t}, \quad \eta=\frac{y}{t}, \quad \gamma=\frac{z}{t}, \quad \xi_{o}=\frac{a}{t}, \quad B i=\frac{h_{c} t}{k}, \quad \eta_{o}=\frac{b}{t}, \quad \alpha=\frac{\sigma_{o}}{t}, \quad \beta=\frac{\sigma_{z}}{t},
$$


and the rest of the surfaces are insulated (Fig. 1.b). The general solution yields

$$
\bar{T}=A_{o}\left(\gamma-1-\frac{1}{B i}\right)+\sum_{m=0}^{\infty} \sum_{n=0}^{\infty} A_{m n} \cos \left(\frac{2 m \pi \xi}{\xi_{o}}\right) \cos \left(\frac{2 n \pi \eta}{\eta_{o}}\right) Z_{m n}(\gamma)
$$

where

$$
\begin{aligned}
& A_{o}=-\frac{8 \alpha \beta \sqrt{\pi}}{3 \xi_{\circ} \eta_{o}} \operatorname{erf}\left(\frac{\xi_{o}}{2 \sqrt{2} \alpha}\right) \\
& A_{m n}=-\frac{2 \sqrt{2 \pi} \alpha \operatorname{Re}\left[\operatorname{erf}\left(\frac{\sqrt{2} \xi_{o}^{2}-4 \sqrt{2} i \pi \alpha^{2} m}{4 \alpha \xi_{o}}\right)\right]}{\left.\exp \left(\frac{2 \pi^{2} \alpha^{2} m^{2}}{\xi_{o}^{2}}\right) \xi_{\circ} \eta_{o} \lambda_{m n} \frac{d Z_{m n}}{d \gamma}\right|_{\gamma=0}}\left[\frac{2 \eta_{o}^{3} \sin \left(\frac{\sqrt{2} \beta 2 n \pi}{\eta_{o}}\right)}{\beta^{2}(2 n \pi)^{3}}-\frac{2 \sqrt{2} \eta_{o} \cos \left(\frac{\sqrt{2} \beta 2 n \pi}{\eta_{o}}\right)}{\beta(2 n \pi)^{2}}\right], \\
& Z_{m n}(\gamma)=\exp \left(-\lambda_{m n} \gamma\right)\left[1+\frac{\lambda_{m n}-B i}{\lambda_{m n}+B i} \exp \left(2 \lambda_{m n}(\gamma-1)\right)\right]
\end{aligned}
$$

and

$$
\lambda_{m n}=\sqrt{\left(\frac{2 m \pi}{\xi_{o}}\right)^{2}+\left(\frac{2 n \pi}{\eta_{o}}\right)^{2}} .
$$

The temperature is applicable to photon shutters and fixed masks. Note that due to the slow convergence of the double infinity series in Eq. 17, at least 500 terms for each index is required.

\section{Infinity Wedge, $0 \leq \theta \leq \theta_{o}$ with Gaussian Power Heating along $\theta=0$ with Peak Power at the Apex}

The governing equation reads

$$
D\left(\frac{\partial^{2} T}{\partial r^{2}}+\frac{1}{r} \frac{\partial T}{\partial r}+\frac{1}{r^{2}} \frac{\partial^{2} T}{\partial \theta^{2}}+\frac{\partial^{2} T}{\partial z^{2}}\right)+\frac{Q}{\rho c}=\frac{\partial T}{\partial t}
$$

where $D=\frac{k}{\rho c}$, and the boundary conditions are assumed to be

$$
\frac{\partial T}{\partial \theta}(r, 0)=\frac{\partial T}{\partial \theta}\left(r, \theta_{o}\right)=0
$$

and

$$
Q(r, \theta)=q_{o} \exp \left(-\frac{r^{2}}{2 \sigma_{o}^{2}}\right) \delta(\theta)
$$

The geometry is shown in Fig. 1.c. The solution can be written in integral form:

$$
T=\frac{q_{0}}{\rho c} \int_{0}^{t} d t^{\prime} \int_{0}^{\infty} r^{\prime} d r^{\prime} \int_{0}^{\theta_{0}} d \theta^{\prime} \int_{-\infty}^{\infty} d z^{\prime} Q\left(r^{\prime}, \theta^{\prime}\right) G^{*}
$$


where $G^{*}$ is the Green function (or Influence function, Fundamental solution ) given as [7]

$$
G^{*}=\frac{\left(D\left(t^{\prime}-t\right)\right)^{-\frac{3}{2}}}{4 \theta_{o} \sqrt{\pi}} \exp \left(-\frac{z^{2}+r^{2}+{r^{\prime}}^{2}}{4 D\left(t-t^{\prime}\right)}\right)\left[I_{o}\left(\frac{r r^{\prime}}{2 D\left(t-t^{\prime}\right)}\right)+2 \sum_{n=1}^{\infty} \cos (s \theta) \cos \left(s \theta^{\prime}\right) I_{s}\left(\frac{r r^{\prime}}{2 D\left(t-t^{\prime}\right)}\right)\right]
$$

for this particular geometry and boundary conditions. $s=\frac{n \pi}{\theta_{o}}$ and $I_{s}(x)$ is the modified Bessel function of order $s$. Substituting Eq. (24) into Eq. (23), after some algebra the temperature can be expressed as

$$
T=\frac{q_{o} \sqrt{\pi}}{2 \rho c \sqrt{2} \sigma_{o} \theta_{o}} \int_{o}^{t} d t^{\prime} \frac{1}{\sqrt{\tau_{2}}} \exp \left(-\frac{r^{2}}{2 \sigma_{o}^{2}\left(1+\tau_{1}\right)}\right)\left[I I_{o}\left(\frac{r^{2}}{4 \sigma_{o}^{2} \tau_{2}}\right)+2 \sum_{n=1}^{\infty} \cos (s \theta) I_{\frac{s}{2}}\left(\frac{r^{2}}{4 \sigma_{o}^{2} \tau_{2}}\right)\right]
$$

where

$$
\tau_{1}=\frac{4 D\left(t-t^{\prime}\right)}{2 \sigma_{o}^{2}}, \quad \tau_{2}=\frac{4 D\left(t-t^{\prime}\right)}{2 \sigma_{o}^{2}}\left(1+\frac{4 D\left(t-t^{\prime}\right)}{2 \sigma_{o}^{2}}\right)=\tau_{1}\left(1+\tau_{1}\right)
$$

and the function $I I_{\nu}(x)$ is the scaled modified Bessel function defined by

$$
I I_{\nu}(x)=\exp (-x) I_{\nu}(x)
$$

Note that for the semi-infinite plane $\theta_{0}=\pi$, the solution to Eq. (25) can be written as

$$
T=\frac{q_{o}}{2 \sqrt{2 \pi} \rho c \sigma_{o}} \int_{o}^{t} \frac{1}{\sqrt{\tau_{2}}} \exp \left(\frac{r^{2} \cos ^{2} \theta}{2 \sigma_{o}^{2} \tau_{2}}-\frac{r^{2}}{2 \sigma_{o}^{2} \tau_{1}}\right)\left[1+\operatorname{erf}\left(\frac{r \cos \theta}{\sqrt{2} \sqrt{\tau_{2}} \sigma_{o}}\right)\right] .
$$

If another half of the Gaussian power is placed on $\theta=\theta_{0}=\pi$, this results in a semi-infinite plane subjected to an entire Gaussian heat power. The resulting solution yields

$$
T=\frac{q_{o}}{\sqrt{2 \pi} \rho c \sigma_{o}} \int_{o}^{t} d t^{\prime} \frac{1}{\sqrt{\tau_{2}}} \exp \left(-\frac{x^{2}}{2 \sigma_{o}^{2}\left(1+\tau_{1}\right)}-\frac{y^{2}}{2 \sigma_{o}^{2} \tau_{1}}\right)
$$

and the maximum temperature can be expressed as

$$
T=\frac{2 \sigma_{o} q_{o}}{4 \sqrt{2 \pi} D \rho c} \log \left(2 \sqrt{\left(\frac{4 D t}{2 \sigma_{o}^{2}}\right)^{2}+\left(\frac{4 D t}{2 \sigma_{o}^{2}}\right)}+\frac{4 D t}{\sigma_{o}^{2}}+1\right),
$$

which is identical to the results described in [3].

If a Gaussian-Parabolic type of heating power is applied on the infinite wedge $\left(0 \leq r \leq \infty, 0 \leq \theta \leq \theta_{0},-\infty \leq\right.$ $z \leq \infty)$ where the parabolic power profile is along the off-plane direction, then $Q$ in Eq. (20) is rewritten as

$$
Q=q_{o} \exp \left(-\frac{r^{2}}{2 \sigma_{o}^{2}}\right)\left(1-\frac{z^{2}}{2 \sigma_{z}^{2}}\right) \delta(\theta)
$$

and the additional temperature field at instant time $t^{\prime}$ is

$$
v=\int_{-\infty}^{\infty} d z^{\prime} \frac{1}{2 \sqrt{\pi D\left(t-t^{\prime}\right)}} \exp \left(-\frac{\left(z-z^{\prime}\right)^{2}}{4 D\left(t-t^{\prime}\right)}\right)\left(1-\frac{z^{\prime 2}}{2 \sigma_{z}^{2}}\right)=\left[1-\frac{D\left(t \cdots t^{\prime}\right)}{\sigma_{z}^{2}}\left(1+\frac{2 z^{2}}{4 D\left(t-t^{\prime}\right)}\right)\right] .
$$

Multiplying Eq. (25) by Eq. (28), the temperature becomes

$$
\begin{aligned}
T= & \frac{q_{o} \sqrt{\pi}}{2 \sqrt{2} \sigma_{o} \theta_{o} \rho c} \int_{o}^{t} d t^{\prime} \frac{1}{\sqrt{\tau_{2}}} \exp \left(-\frac{r^{2}}{2 \sigma_{o}^{2}\left(1+\tau_{1}\right)}\right) \times \\
& {\left[I I_{o}\left(\frac{r^{2}}{4 \sigma_{o}^{2} \tau_{2}}\right)+2 \sum_{n=1}^{\infty} \cos (s \theta) I I_{\frac{s}{2}}\left(\frac{r^{2}}{4 \sigma_{o}^{2} \tau_{2}}\right)\right]\left[1-\frac{D\left(t-t^{\prime}\right)}{\sigma_{z}^{2}}\left(1+\frac{2 z^{2}}{4 D\left(t-t^{\prime}\right)}\right)\right] . }
\end{aligned}
$$


The two-dimensional temperature solution in Eq. (25) can be applied to bending magnet beam missteering heating at the wedge of the Aluminum positron chamber whereas the three-dimensional temperature solution in Eq. (33) can be applied to undulator/wiggler beam missteering heating. Figure 3.a shows the temperature contours of the wedge heated by half of the Gaussian power at 60 seconds when $\theta_{0}=\frac{5 \pi}{6}$, $q_{o}=17.16 \frac{\mathrm{W}}{\mathrm{mm}^{2}}$, and $\sigma_{o}=0.16 \mathrm{~mm}$. The Gaussian power is applied along the $x$ axis where the center of the flux is at the origin. The maximum temperature rise shown in the contours is about $66{ }^{\circ} \mathrm{C}$ and is not located at the origin. Since the other side of the wedge is not heated and the heat flow can travel along that

- direction, the temperature at the origin is reduced. Figure 3.b shows the temperature rise due to different wedge angles $\theta_{o}$. It is found that when $t>0.2$ seconds, temperature rise is inversely proportional to the wedge angle. This is because a larger wedge angle indicates a larger area, which diffuses the heat and reduces the temperature more efficiently.

\section{Thin Disk, $0 \leq r \leq r_{o}$, with Axisymmetrical Gaussian Power Heating on One Side and Cooling on the Other Side}

As shown in Fig. 1.d, the disk is so thin that the power flow and convective cooling can be implemented as heat source and sink terms in the heat equation, and the governing equation can be written as [7]

$$
\frac{\partial^{2} T}{\partial r^{2}}+\frac{1}{r} \frac{\partial T}{\partial r}-\frac{h_{c}}{k t}\left(T-T_{\infty}\right)+\frac{q_{o}}{k t} \exp \left(-\frac{r^{2}}{2 \sigma_{o}^{2}}\right)=0
$$

and the boundary conditions

$$
\begin{aligned}
& \frac{\partial T}{\partial r}=0, \quad \text { on } r=r_{\circ} \\
& |T|=\text { finite, } \quad \text { on } r=0
\end{aligned}
$$

are used. The following solution is obtained

$$
T-T_{\infty}=A I_{o}(R)+u(R)
$$

where $R$ is defined as

$$
R=r \sqrt{\frac{h_{c}}{k t}},
$$

$A$ is the undetermined coefficient, and $I_{o}$ is the modified Bessel function of the zero kind. $u(R)$ is the particular solution given as [8]

$$
u(R)=I_{o}(R) \int_{0}^{R} \frac{1}{\tau} K_{o}(\tau) f(\tau) d \tau-K_{o}(R) \int_{0}^{R} \frac{1}{\tau} I_{o}(\tau) f(\tau) d \tau
$$

where

$$
f(r)=-\frac{q_{o} t}{h_{c}} r^{2} \exp \left(-\frac{k t r^{2}}{4 h_{c} \sigma_{o}^{2}}\right) .
$$

Substituting Eqs. (39) and (37) into the boundary condition Eq. (35), the coefficient $A$ can be obtained

$$
I_{1}\left(R_{o}\right) A=-I_{o}\left(R_{o}\right) \int_{o}^{R_{o}} \frac{1}{\tau} K_{o}(\tau) f(\tau) d \tau+K_{o}\left(R_{o}\right) \int_{o}^{R_{o}} \frac{1}{\tau} I_{o}(\tau) f(\tau) d \tau
$$

where $R_{o}=\sqrt{\frac{H}{K D}} r_{o}$. Note that the coefficient $A$ is the maximum temperature as $R \rightarrow 0$.

The result can be applied to monochromators or Aluminum windows in the linac. For example, if the $\mathrm{Al}$ window is $0.05 \mathrm{~mm}$ thick, it absorbs $1 \%$ of the total power, if $0.1 \mathrm{~mm}$ thick, then $2 \%$ is absorbed and so forth. For a given distance, the beam size is found to be $5 \mathrm{~mm}$ in diameter and the total electron power is $500 \mathrm{~W}$. The back side of the window is subjected to air cooling. Fig. 4 shows the maximum temperature isotherm verses the window thickness and air convection coefficient. The current design with $t=2$ mills, $h_{c}=i \frac{W}{m^{2}{ }^{\circ} \mathrm{C}}$, gives a maximum temperature rise of $345{ }^{\circ} \mathrm{C}$. 


\section{Beryllium -Copper Composite, $0 \leq x \leq a,-b \leq y \leq b$, Gaussian Heat Generation on Beryllium, Surface Heating on Beryllium-Copper Interface, Cooling on Back Side of the Copper Surface}

As shown in Fig. 1.e, a beryllium block is heated by a Gaussian-type x-ray and a copper block is joined with the beryllium. Water cooling is supplied on the back of the copper. Since the $\mathrm{x}$-ray is able to transmit into the beryllium material, it is simulated as a heat generation in beryllium. On the other hand, the rest of the - power is deposited on the beryllium-copper interface. Therefore, the governing equations become

$$
\frac{\partial^{2} T_{C u}}{\partial x^{2}}+\frac{\partial^{2} T_{C u}}{\partial y^{2}}=0
$$

and

$$
\frac{\partial^{2} T_{B e}}{\partial x^{2}}+\frac{\partial^{2} T_{B e}}{\partial y^{2}}=-\frac{q_{o}}{k_{B e}} \exp \left(-\frac{y^{2}}{2 \sigma_{o}^{2}}\right) \alpha_{t} \exp \left(-\alpha_{t}|x|\right)
$$

with the boundary conditions

$$
\begin{array}{ll}
\frac{\partial T_{B e}}{\partial y}=\frac{\partial T_{C u}}{\partial y}=0, & \text { on } y= \pm b \\
T_{B e}=T_{C u}, & \text { on } x=0 \\
k_{C u} \frac{\partial T_{C u}}{\partial x}-k_{B e} \frac{\partial T_{B e}}{\partial x}=-q_{o} \exp \left(-\frac{y^{2}}{2 \sigma_{o}^{2}}\right) \exp \left(-\alpha_{t} a_{1}\right), \text { on } x=0 \\
k_{B e} \frac{\partial T_{B e}}{\partial x}=0, & \text { on } x=-a_{1} \\
k_{C u} \frac{\partial T_{C u}}{\partial x}=-h_{c}\left(T_{C u}-T_{\infty}\right), & \text { on } x=a_{2},
\end{array}
$$

The boundary conditions suggest that the temperature solutions must have the form of

$$
T_{C u}-T_{\infty}=\left(X_{C u}\right)_{o}(x)+\sum_{m=1}^{\infty}\left(X_{C u}\right)_{m}(x) \cos \lambda_{m} y, \quad T_{B e}-T_{\infty}=\left(X_{B e}\right)_{o}(x)+\sum_{m=1}^{\infty}\left(X_{B e}\right)_{m}(x) \cos \lambda_{m} y(46)
$$

where $\lambda_{m}=\frac{m \pi}{b}$, and the associated coefficients satisfy

$$
\begin{array}{lc}
\frac{\partial^{2}\left(X_{C u}\right)_{o}}{\partial x^{2}}=0, & \frac{\partial^{2}\left(X_{B e}\right)_{o}}{\partial x^{2}}=\alpha_{o} \alpha_{t} \frac{\exp \left(-\alpha_{t} x\right)}{k_{B e}}, \\
\frac{\partial^{2}\left(X_{C u}\right)_{m}}{\partial x^{2}}-\lambda_{m}^{2}\left(X_{C u}\right)_{m}=0, & \frac{\partial^{2}\left(X_{B e}\right)_{m}}{\partial x^{2}}-\lambda_{m}^{2}\left(X_{B e}\right)_{m}=\frac{\alpha_{m} \alpha_{t}}{k_{B e}} \exp \left(-\alpha_{t}|x|\right),
\end{array}
$$

where

$$
\begin{aligned}
& \alpha_{o}=\frac{1}{2 b} \int_{-b}^{b} q_{o} \exp \left(-\frac{y^{2}}{2 \sigma_{o}^{2}}\right) d y=\frac{q_{o} \sqrt{2 \pi} \sigma_{o}}{2 b} \operatorname{erf}\left(\frac{\sqrt{2} b}{2 \sigma_{o}}\right) \\
& \alpha_{m}=\frac{1}{b} \int_{-b}^{b} q_{o} \exp \left(-\frac{y^{2}}{2 \sigma_{o}^{2}}\right) \cos \lambda_{m} y d y=\frac{q_{o} \sqrt{2 \pi} \sigma_{o}}{b} \exp \left(-\frac{\lambda_{m}^{2} \sigma_{o}^{2}}{2}\right) \operatorname{Re}\left[\operatorname{erf}\left(\frac{\sqrt{2} b+i \sqrt{2} \lambda_{m} \sigma_{o}^{2}}{2 \sigma_{o}}\right)\right] .
\end{aligned}
$$


The general solutions in Eq. (46) are obtained as follows :

$$
\begin{aligned}
& \left(X_{C u}\right)_{o}=A_{o} x+B_{o} \\
& \left(X_{B e}\right)_{o}=C_{o} x+B_{o}+\frac{\alpha_{o}}{\alpha_{t} k_{B e}} \exp \left(-\alpha_{t} x\right) \\
& \left(X_{C u}\right)_{m}=A_{m} \cosh \lambda_{m} x+B_{m} \sinh \lambda_{m} x \\
& \left(X_{B e}\right)_{m}=C_{m} \cosh \lambda_{m} x+D_{m} \sinh \lambda_{m} x-\frac{\alpha_{m} \alpha_{t}}{k_{B e}\left(\lambda_{m}^{2}-\alpha_{t}^{2}\right)} \exp \left(-\lambda_{t}|x|\right) .
\end{aligned}
$$

Substituting Eq. (48) into Eq. (44), one finds

$$
A_{o}=\frac{\alpha_{o}}{k_{C u}}, B_{o}=-\frac{\alpha_{o}\left(a_{2} h+k_{C u}\right)}{h k_{C u}}, C_{o}=\frac{\alpha_{o}}{k_{B c}}, D_{o}=\alpha_{o}\left(\frac{1}{h}+\frac{a_{2}}{k_{C u}}+\frac{1}{\alpha_{t} k_{B e}} \exp \left(-\alpha_{t} a_{1}\right)\right)
$$

and all coefficients $A_{m}, B_{m}, C_{m}$ and $D_{m}$ satisfy the following system equations :

$$
\begin{aligned}
& A_{m}\left(K_{C u} \lambda_{m} \sinh \lambda_{m} a+h \cosh \lambda_{m} a\right)+B_{m}\left(K_{C u} \lambda_{m} \cosh \lambda_{m} a+h \sinh \lambda_{m} a\right)=0 \\
& A_{m} \cosh \lambda_{m} a_{1}+B_{m} \sinh \lambda_{m} a_{1}-C_{m} \cosh \lambda_{m} a_{1}-D_{m} \sinh \lambda_{m} a_{1}=-\frac{\lambda_{m} \alpha_{t}}{k_{B e}\left(\lambda_{m}^{2}-\alpha_{t}^{2}\right)} \\
& A_{m} k_{C u} \lambda_{m} \sinh \lambda_{m} a_{1}+B_{m} k_{C u} \lambda_{m} \cosh \lambda_{m} a_{1}- \\
& \quad C_{m} k_{B e} \lambda_{m} \sinh \lambda_{m} a_{1}-D_{m} k_{B e} \lambda_{m} \cosh \lambda_{m} a_{1}=\frac{\lambda_{m}^{2} \alpha_{m}^{2}}{\lambda_{m}^{2}-\alpha_{t}^{2}} \exp \left(-\alpha_{t} a_{1}\right) \\
& D_{m} \lambda_{m}=-\frac{\alpha_{m} \alpha_{t}^{2}}{k_{B e}\left(\lambda_{m}^{2}-\alpha_{t}^{2}\right)} .
\end{aligned}
$$

Note that the system equation in Eq. (50) is better solved numerically. At least 100 terms are required to meet the convergence value.

The type-B design cylindrical absorber in APS [3], a $\frac{1}{8}$ inch beryllium ring, is brazed on a hollow copper cylinder through which water runs vertically to remove the heat. The area where the bending magnet beam hits at normal incident is the place to be optimized. Since the photon fan strikes horizontally and the circumference of the crotch absorber is much larger than the vertical beam size, the temperature solution in Eq. (45) is applicable to the situation. Figures 5, 6, and 7 show the parametric studies of the temperature at the beryllium heating surface (point A in Fig. 1.e), beryllium-copper interface (point B in Fig. 1.e), and copper tube (point C in Fig. 1.e) temperatures, respectively. The total height is $\frac{3}{8}$ inch, $q_{o}=120 \frac{\mathrm{W}}{\mathrm{m}^{2}}$, and $\sigma_{o}=0.11 \mathrm{~m}$ at $100 \mathrm{~mA}$. The water convection coefficient is set to $2 \frac{\mathrm{W}}{\mathrm{m}^{2} \mathrm{o}_{\mathrm{C}}}$ and $\alpha_{t}=53.02 \frac{1}{\mathrm{~m}}$ is chosen [2]. The horizontal axes in the figures indicate the change of the beryllium thickness whereas the vertical axes show total beryllium-copper thickness. In Figure 5 the maximum beryllium surface temperature car be minimized at about $160^{\circ} \mathrm{C}$ if 0.075 inch $<a$ (total beryllium-copper thickness) $<0.2$ inch and 0.125 inch $<a_{1}$ (beryllium thickness) $<0.25$ inch. But as shown in Fig. 6, the optimized maximum beryllium-copper interface temperature is not found. The lowest temperature occurred when $a \approx 0.25$ inch and $a_{1} \approx 0.2$ inch, which is approximately $155^{\circ} \mathrm{C}$. On the other hand, the maximum copper-water interface temperature is almost proportional to the beryllium thickness (Fig. 7). This analysis, however, provides a state-of-the-art parametric analysis for use in designing beryllium-copper composite absorbers.

\section{Acknowledgement}

The authors would like to thank Ms. Catherine Eyberger for editing this paper.

\section{References}

[1] M. Choi. A Study of Heat Transfer for Two Layered Composite Inclined Plate Crotch Absorbers. Argonne National Laboratory Light Source Note LS-143, 1989. 
[2] M. Choi. The Effects of Photon Spectrum and Variable Thermal Conductivity on the Distribution of Temperature in an Inclined Plate Crotch Absorber. Argonne National Laboratory Light Source Note LS-144 1989.

[3] I. C. Sheng and J. Howell. Quasi-static Thermoelastic Analysis for a Semi-infinite Plane Subjected to a Gaussian Heat Source - Beam Missteering of the Storage Ring in the APS. Argonne National Laboratory Light Source Note LS-199.

[4] S. Kim. Distribution of the Synchrotron Radiation from Bending Magnets. Argonne National Laboratory Light Source Note LS-91, 1988.

[5] D. Vaughan. X-Ray Data booklet. Lawernce Berkeley Laboratory, University of California, 1986.

[6] H. L. Nian, I. C. Sheng and T. M. Kuzay. Thermal Analysis of a Photon Shutter for APS Front Ends. Nuclear Instruments and Methods in Physics Research, A319, pp. 197 -206, 1992.

[7] H. S. Carslaw and and J. C. Jaeger. Conduction of Heat in Solids. Oxford University Press, 2nd edition, 1959.

[8] H. Batemaneger. Higher Transcendental Functions. McGraw-Hill Book Company, Inc., 1953.

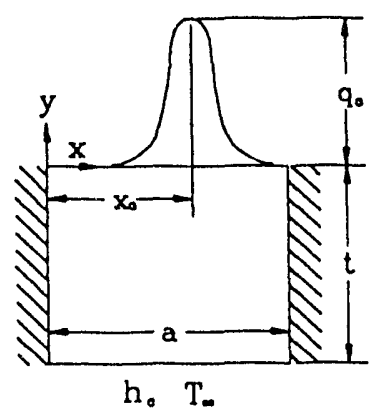

$a$.

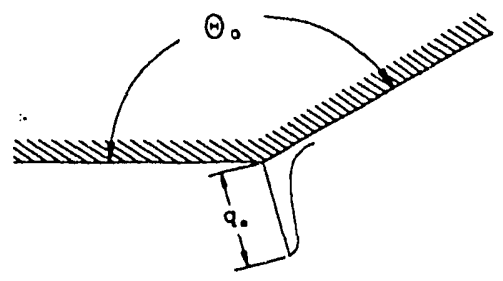

C.

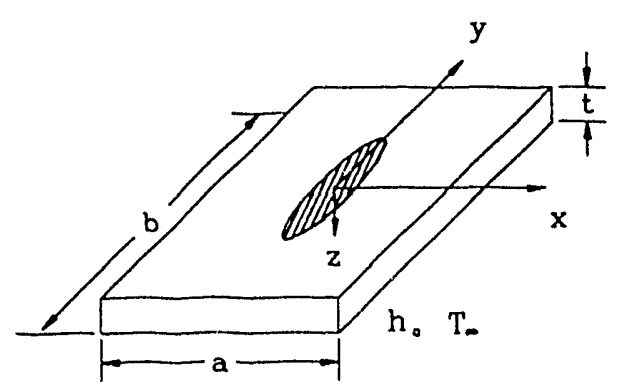

b.

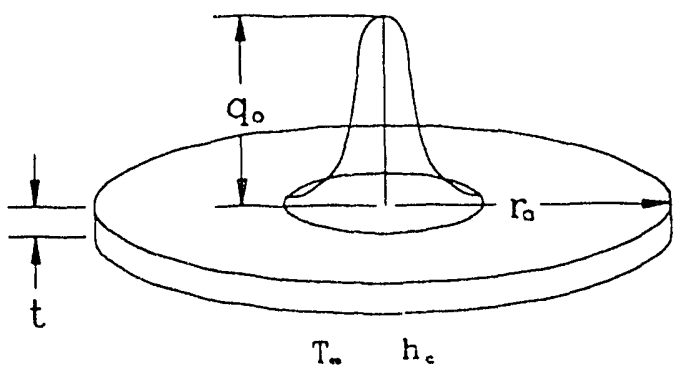

$d$.

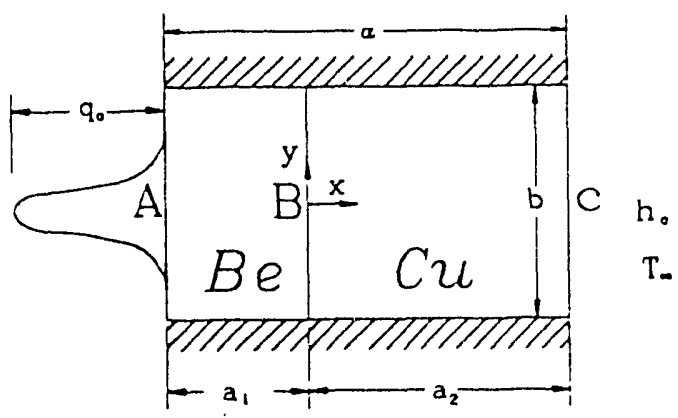

e.

Fig. 1 Power Profiles and Material Geometries 


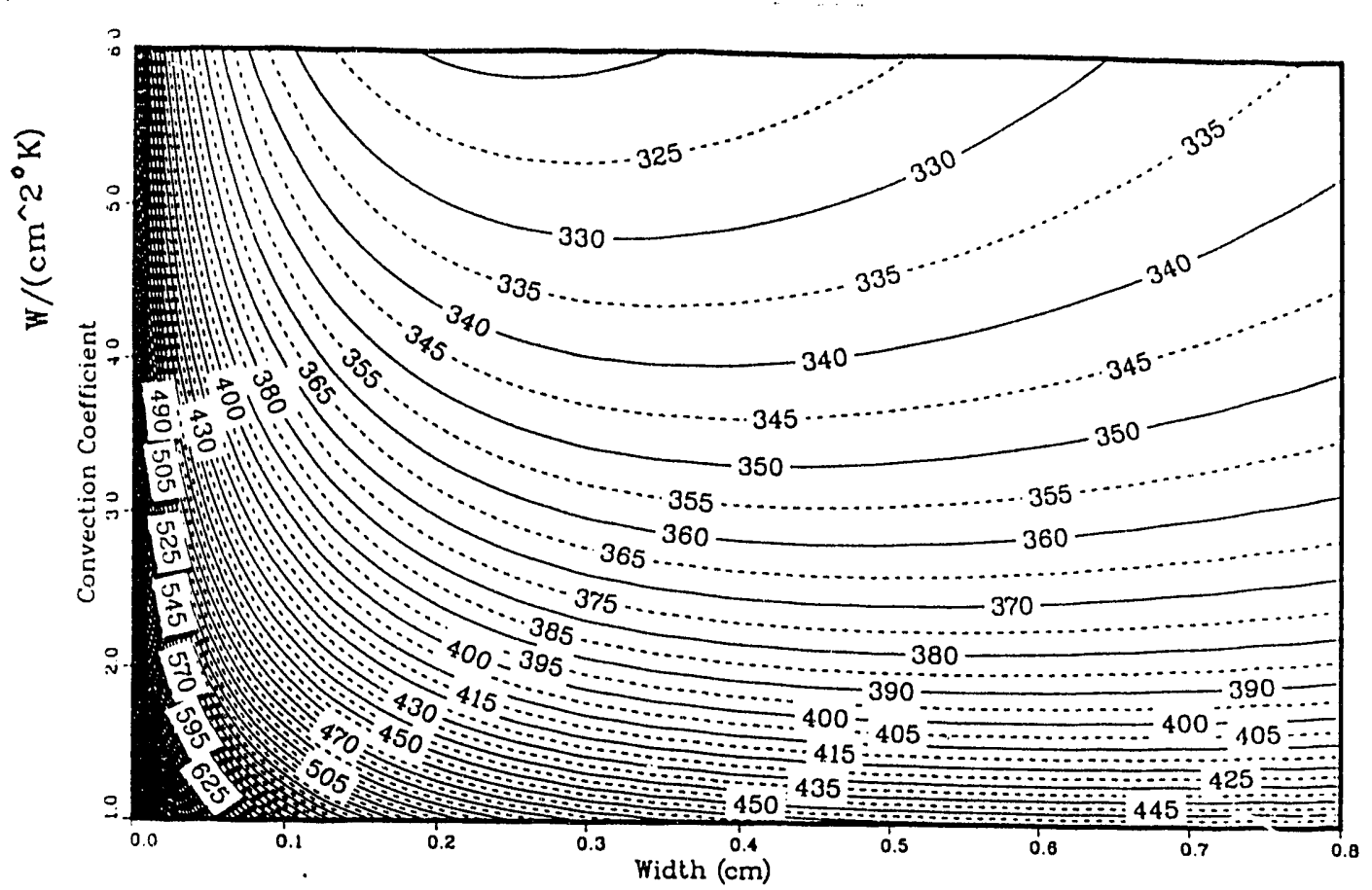

(a)

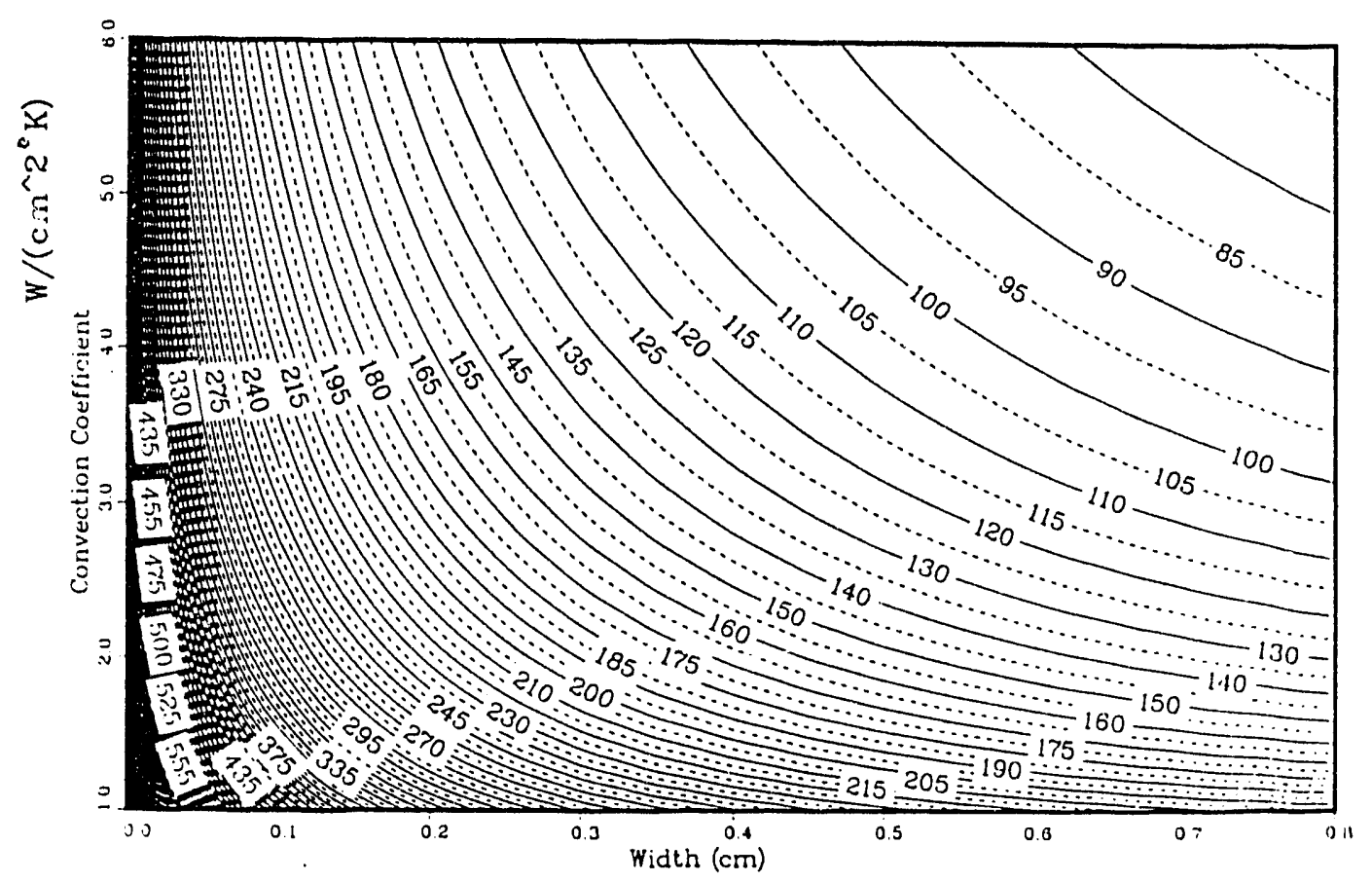

(b)

Fig. 2 Maximum Temperature vs. Width and Convection Coefficient (a) On Heating Surface, (b) Back side of the Cooling Surface 


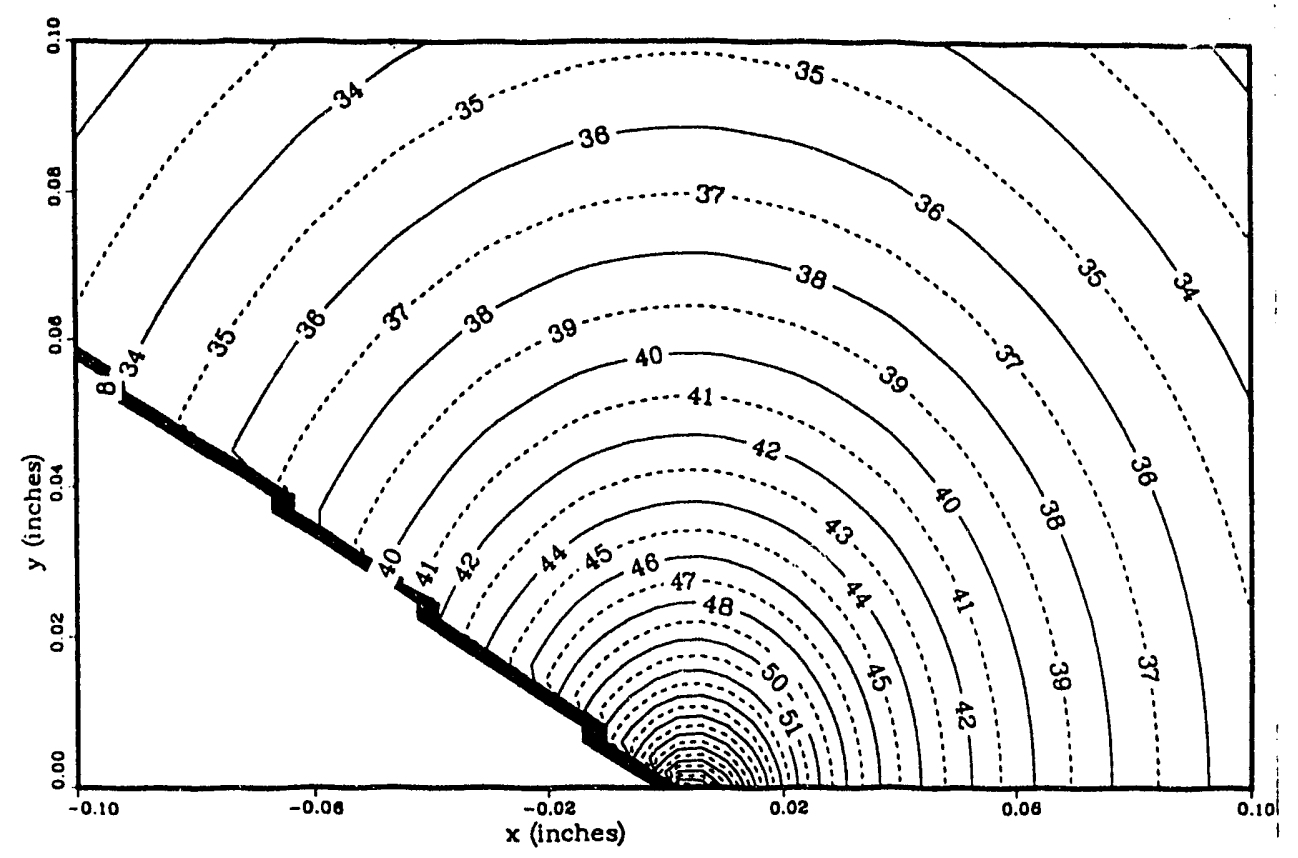

(a)

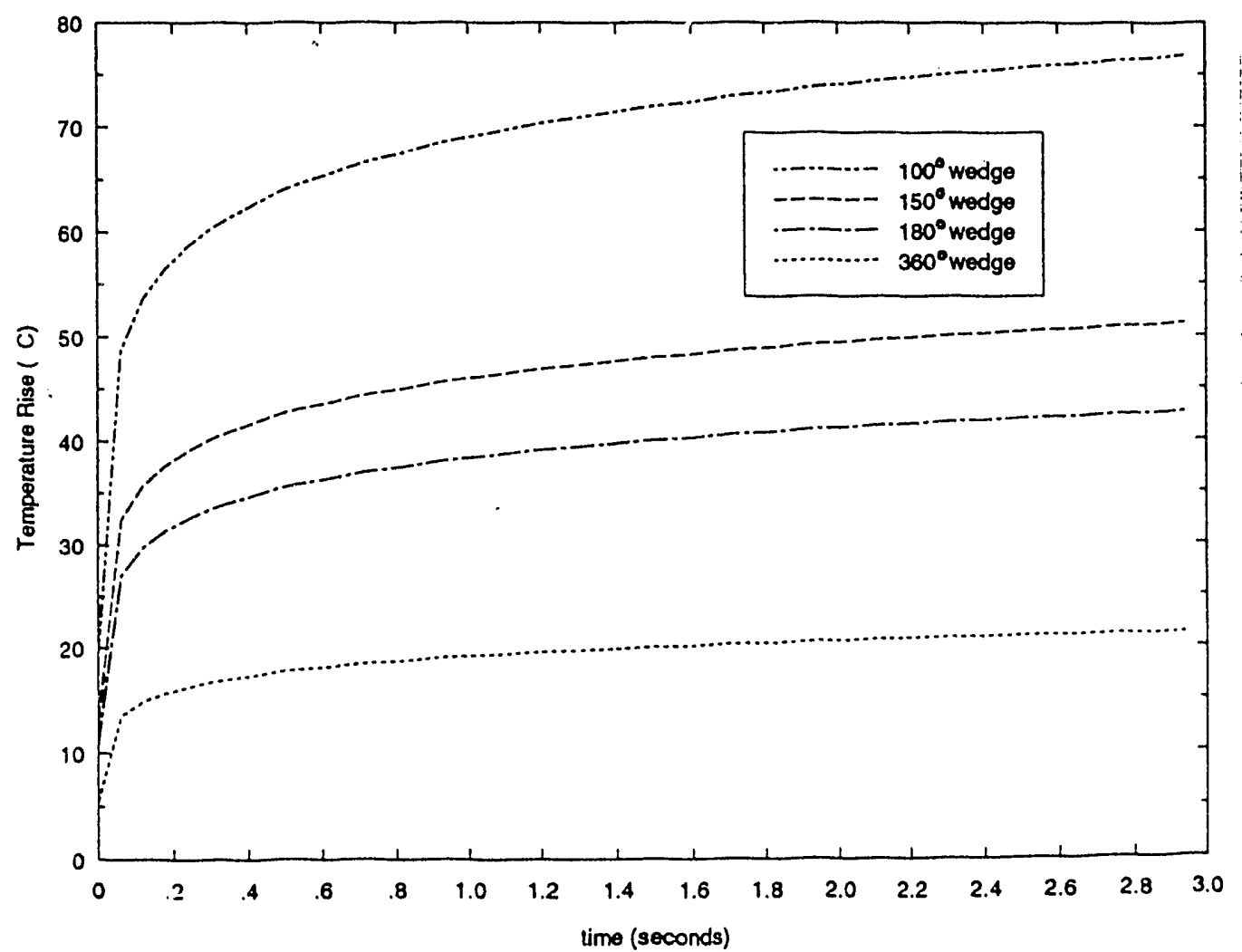

(b)

Fig. 3 Wedge Temperature Profiles (a) Temperature Isotherm, (b) Maximum Temperature Rise for Different Wedge Angles. 


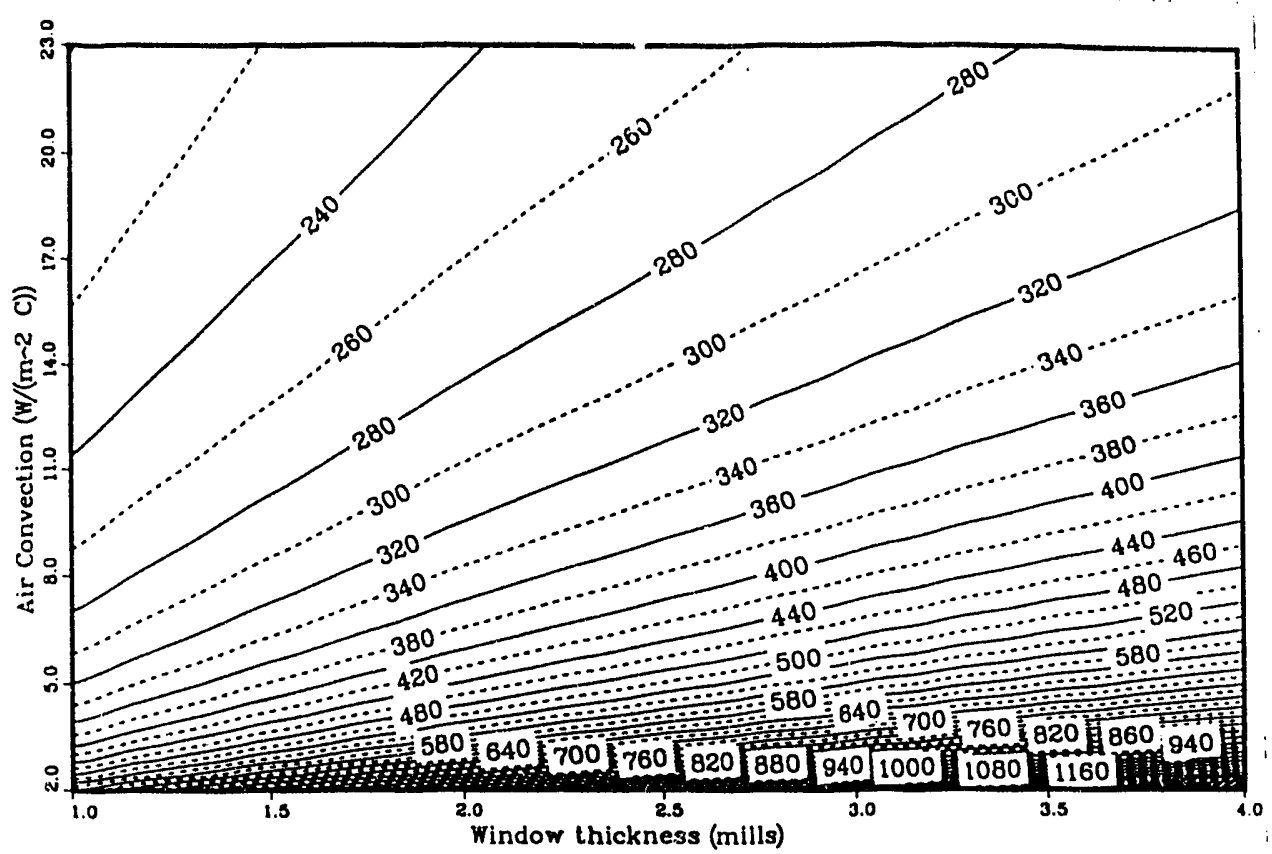

Fig. 4 Maximum Temperature on Al Window vs. Window Thickness and Air Convection Coefficient.

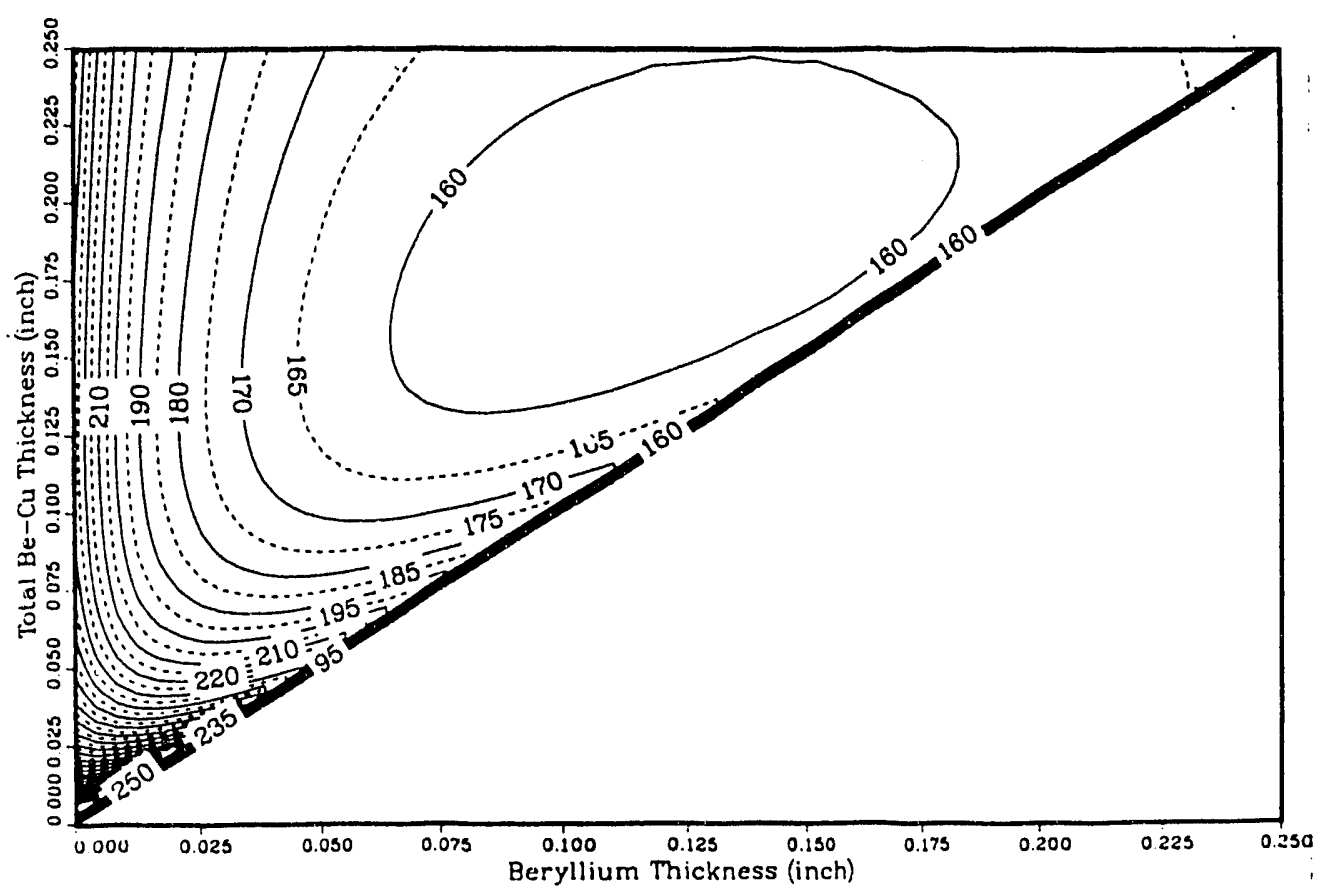

Fig. J Maximum Temperature Rise on Heating Surface of a Beryllium-copper Composite Absorber. Temperature Contours vs. Beryllium Thickness and Total Beryllium-Copper Thickness. 


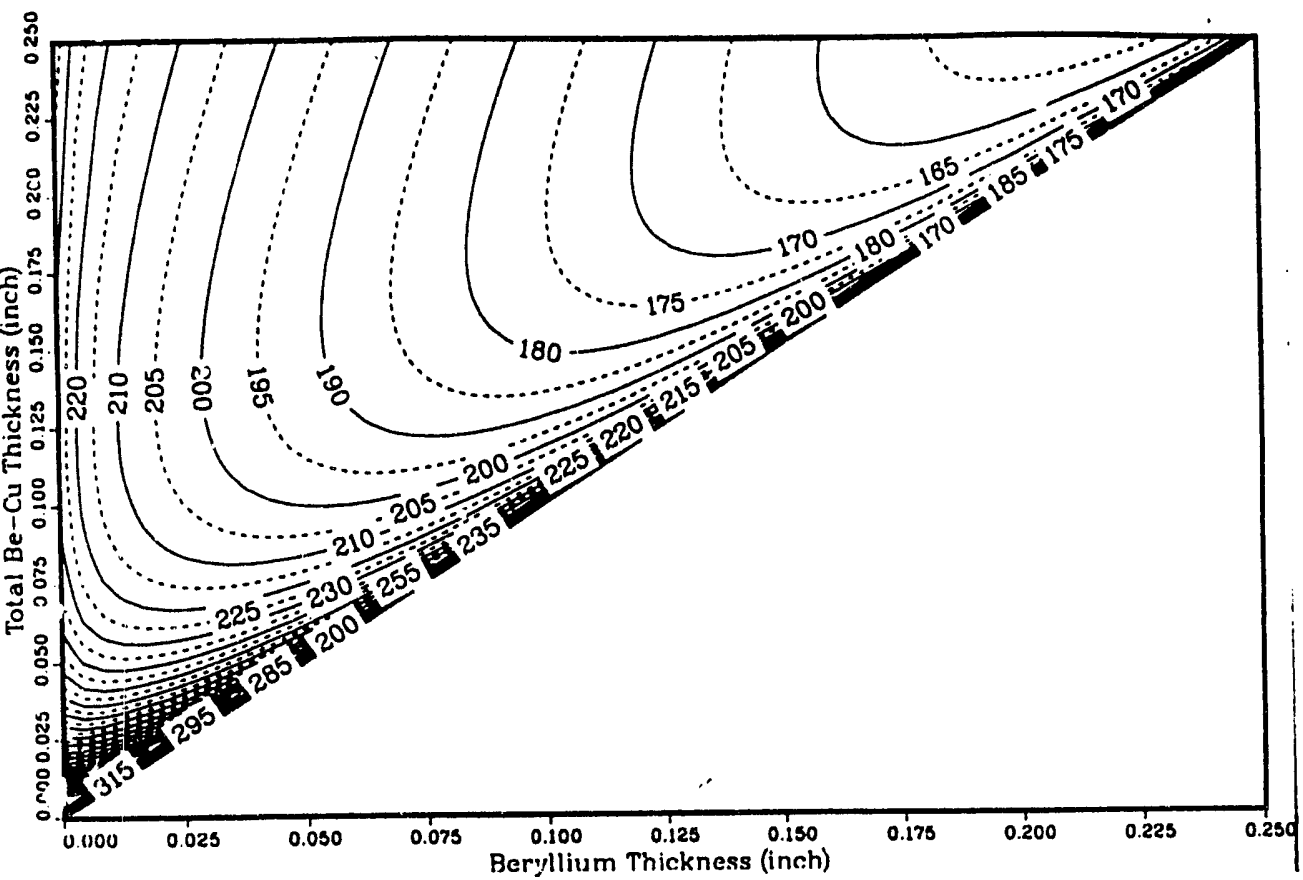

Fig. 6. Maximum Temperature Rise on Interface of a Beryllium-Copper Composite Absorber. Temperature Contours vs. Beryllium Thickness and Total Beryllium-Copper Thickness.

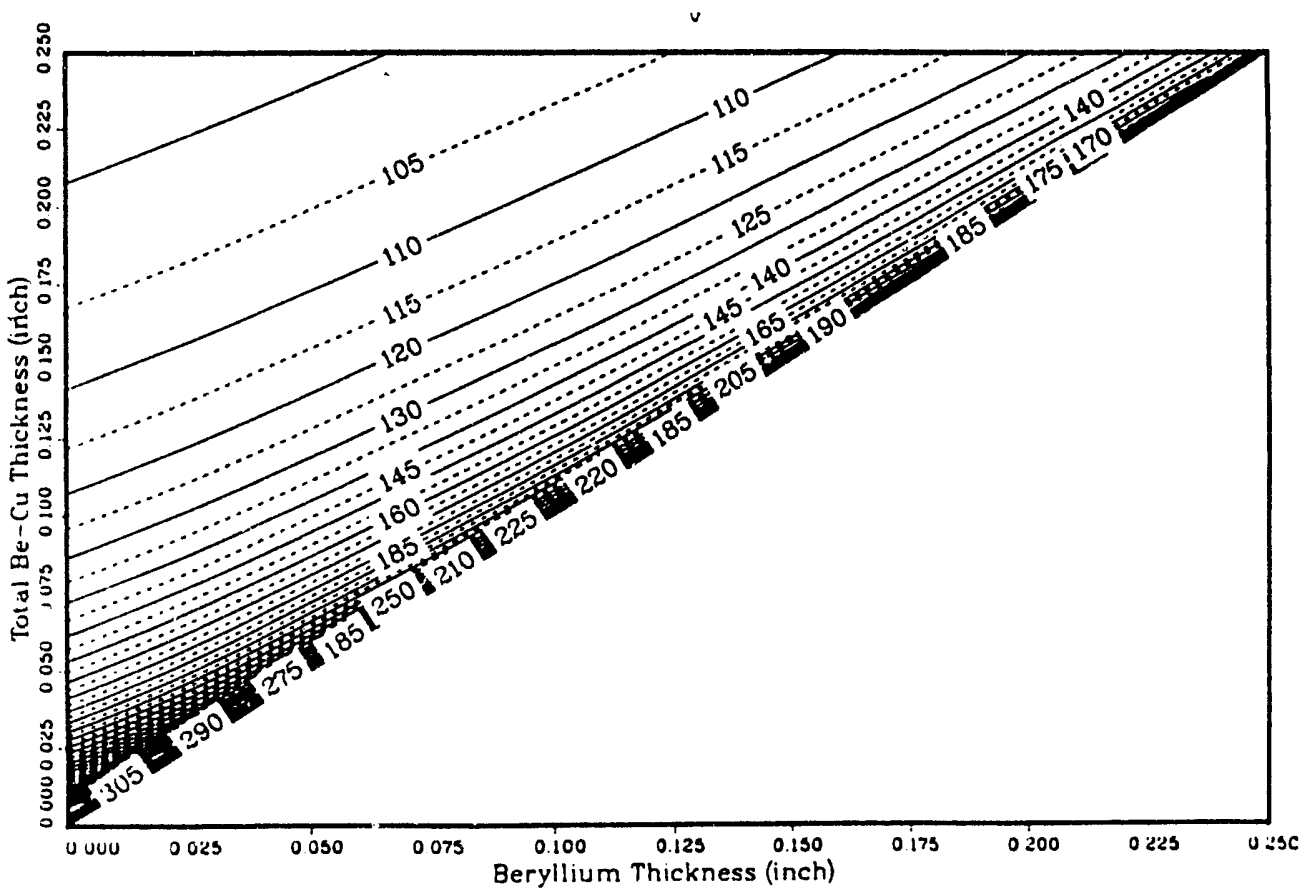

Fig. 7 maximum Temperature Rise on Cooling Surface of a Beryllium-Copper Composite Absorber. Temperature Contours vs. Beryllium Thickness and Total Beryllium-Copper Thickness. 


\section{DISCLAIMER}

This report was prepared as an account of work sponsored by an agency of the United States Government. Neither the United States Government nor any agency thereof, nor any of their employees, makes any warranty, express or implied, or assumes any legal liability or responsibility for the accuracy, completeness, or usefulness of any information, apparatus, product, or process disclosed, or represents that its use would not infringe privately owned rights. Reference herein to any specific commercial product, process, or service by trade name, trademark, manufacturer, or otherwise does not necessarily constitute or imply its endorsement, recommendation, or favoring by the United States Government or any agency thereof. The views and opinions of authors expressed herein do not necessarily state or reflect those of the United States Government or any agency thereof. 

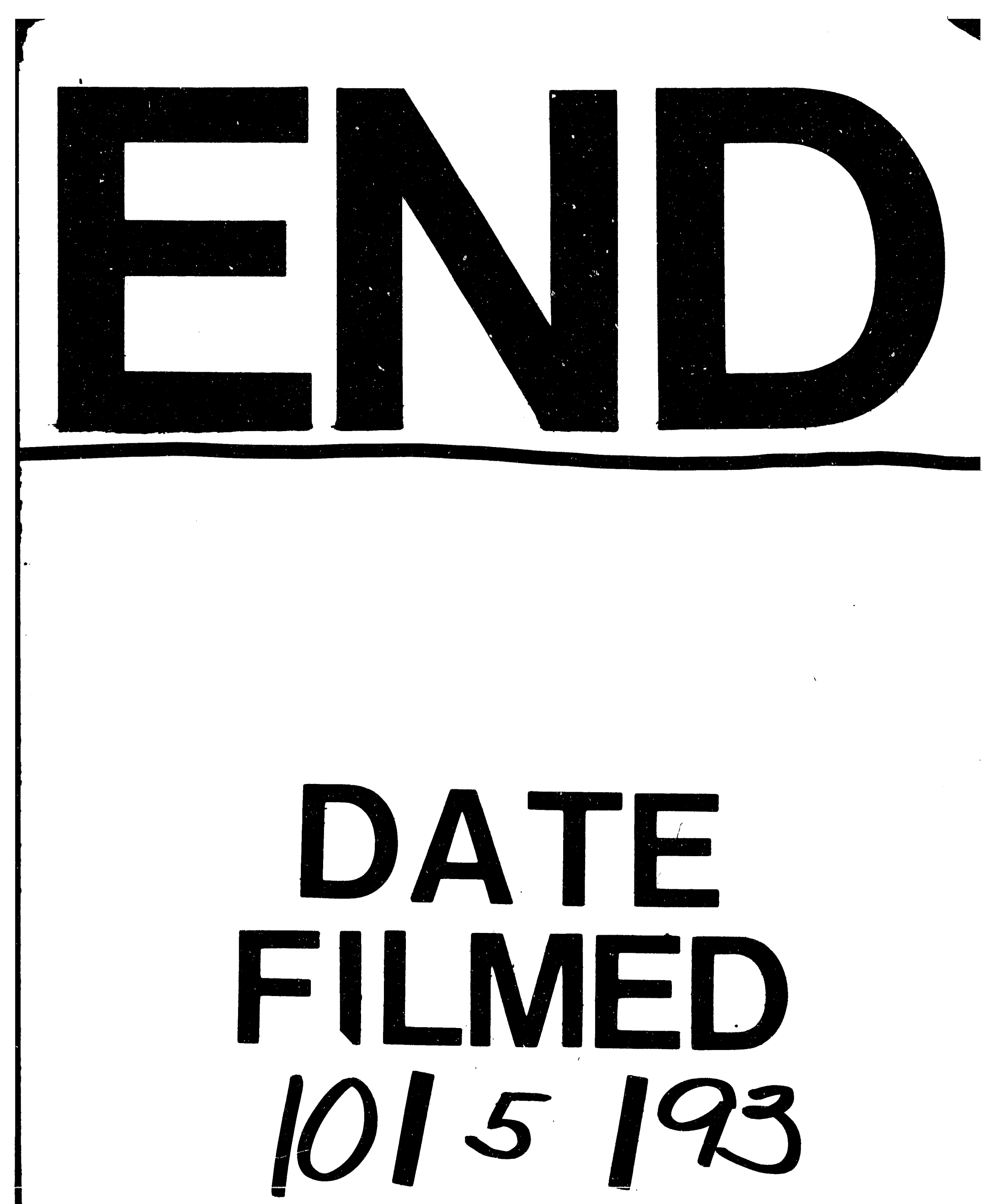
